\title{
Spectral analysis for wide energy channels
}

\author{
E. A. Kronberg and P. W. Daly
}

Max Planck Institute for Solar System Research, Max-Planck-Str. 2, 37191 Katlenburg-Lindau, Germany

Correspondence to: E. A. Kronberg (kronberg@mps.mpg.de)

Received: 22 May 2013 - Published in Geosci. Instrum. Method. Data Syst. Discuss.: 20 August 2013

Revised: 24 October 2013 - Accepted: 28 October 2013 - Published: 18 November 2013

\begin{abstract}
For energetic particle measurements whose spectra follow a power law, it is often challenging to define a characteristic ("effective") energy of an energy channel. In order to avoid time-consuming calculations, the geometric mean is often used as an approximation for the effective energy. This approximation is considered to be pretty good. It is, however, potentially inadequate in cases with wide energy channels and soft spectral slopes. In order to determine the limits of the goodness of the approximation, we derive formulas to calculate the deviation of the effective energy, phase space density and energy density based on the geometric-mean approximation from those based on the power law. The results show that the geometric-mean approximation is usually adequate and that corrections are needed only in extraordinary cases.
\end{abstract}

\section{Introduction}

Particle measurements for the study of space plasma physics are provided in defined energy ranges by instruments onboard satellites. Due to telemetry limitations the energy of each measured particle is not transmitted to the earth. Each particle measurement is allocated to an energy channel with a certain width. Typically, a large number of particles with different energies is recorded by the particle detector at each energy channel. Therefore, it is not straightforward to decide which energy is characteristic for the corresponding energy channel. In practice, to avoid intricate calculations of the effective energy in case of a power law spectral shape, the geometric mean is commonly used for calculations of such physical quantities as, for example, the energy density or the phase space density. In general, this approximation is considered to be pretty good. However, it is not a priori clear to which extent it can be used. For wide energy channels, the approximation is not necessarily always good. Also, soft spectral slopes can lead to significant deviations.

In this paper, we start by showing how the effective energy is derived for a power law spectrum. We then derive formulas that can be used to assess the goodness of the geometricmean approximation for calculating the effective energy, the phase space density and the energy density.

This can be especially helpful for the cases of the wide energy channels at soft power law spectral slopes. This method can be in particular applied to the energetic ion data from the Research with Adaptive Particle Imaging Detector (RAPID) instrument (see Wilken et al., 2001) onboard the Cluster satellites (Escoubet et al., 1997).

\section{Effective energy}

In practice what one measures is the integrated flux $J$ within an energy channel of limits $E_{1}$ and $E_{2}$. The derived mean differential flux $j=J / \Delta E$ then needs to be assigned to some energy value, which we call $E_{\text {eff }}$, the effective energy. For a power law spectrum this value will be

$$
\frac{J}{E_{2}-E_{1}}=j\left(E_{\mathrm{eff}}\right)=A \cdot E_{\mathrm{eff}}^{-\gamma},
$$

where $\gamma$ is a spectral index and $A$ is a normalization. How does one calculate this energy? Here is a simple analysis. We consider a non-relativistical case in this study. The integrated flux is expressed as

$J=\int_{E_{1}}^{E_{2}} A \cdot E^{-\gamma} \mathrm{d} E=\frac{A}{\gamma-1}\left(E_{1}^{-\gamma+1}-E_{2}^{-\gamma+1}\right)$.

Let $E_{\mathrm{m}}=\left(E_{2}+E_{1}\right) / 2, \Delta=\left(E_{2}-E_{1}\right) / 2$ and we denote

$\delta=\frac{\Delta}{E_{\mathrm{m}}}$ 
then Eq. (2) can be transformed to

$$
\begin{aligned}
\frac{J}{E_{2}-E_{1}} & =\frac{A}{\gamma-1} \cdot \frac{(1-\delta)^{-\gamma+1}-(1+\delta)^{-\gamma+1}}{2 \delta} E_{\mathrm{m}}^{-\gamma} \\
& \equiv A \cdot E_{\mathrm{eff}}^{-\gamma} .
\end{aligned}
$$

Equation (4) leads to

$$
\begin{aligned}
\left(\frac{E_{\mathrm{eff}}}{E_{\mathrm{m}}}\right)^{-\gamma} & =\frac{(1-\delta)^{-\gamma+1}-(1+\delta)^{-\gamma+1}}{2 \delta(\gamma-1)} \\
& \simeq 1+\frac{\gamma(\gamma+1) \delta^{2}}{6}+\ldots \\
& \frac{E_{\mathrm{eff}}}{E_{\mathrm{m}}} \simeq 1-\frac{(\gamma+1) \delta^{2}}{6}+\ldots
\end{aligned}
$$

The spectral index $\gamma$ can be estimated by using fluxes for two adjacent energy channels and their effective energies

$\gamma=\frac{\ln \left(j_{1} / j_{2}\right)}{\ln \left(E_{\mathrm{eff}_{2}} / E_{\mathrm{eff}_{1}}\right)}$

Let us look at an extreme case where the upper channel threshold is double the lower one (very wide channel). In this case, where $E_{2}=2 E_{1}, \Delta=E_{1} / 2$, and $E_{\mathrm{m}}=1.5 E_{1}$, we get $\delta=1 / 3$. Requiring $(\gamma+1) \delta^{2} / 6<0.1$ (i.e. $10 \%$ accuracy in $E_{\text {eff }}$, we obtain this accuracy when $\gamma<5.6$, which is usually the case. Thus the mean energy, $E_{\mathrm{m}}$ is a good first approximation of the effective energy, $E_{\text {eff }}$ (i.e. $E_{\text {eff }} \simeq E_{\mathrm{m}}$ ).

Therefore a first guess of $\gamma$ can be obtained from Eq. (6) using $E_{\text {eff }} \simeq E_{\mathrm{m}}$, and then we can get a better estimate of $E_{\mathrm{eff}}$ from the last expression in Eq. (5) and iterate again.

It is also possible to estimate the effective energy in a simpler way assuming for example that $\gamma=4$ then calculate the $E_{\text {eff }}$ using the last expression in formula (5). The estimations of the effective energy at different $\gamma(2-6.5)$ and at $\delta=1 / 3$ show that the values of the effective energy are different from each other to within less than $10 \%$, namely $E_{\text {eff }} / E_{\mathrm{m}} \simeq(0.95-0.86)$. However, for wider channels this method cannot be used.

\section{Effective energy and geometric-mean energy}

One can see that the calculation of the effective energy is either time consuming and for every particle measurement we get different values or if using a simpler approximation it is not applicable for all cases.

An alternative is to use the geometric-mean energy $E_{\mathrm{g}}=\sqrt{E_{1} \cdot E_{2}}$ as an approximation of the effective energy. This method is for example applied to generate a set of fixed conversion factors between differential flux and phase space density for the Cluster/RAPID particle data (see Kronberg and Daly, 2013).

The geometric-mean energy $E_{\mathrm{g}}$ defined above is in fact a good approximation to the effective energy $E_{\text {eff }}$. Let us recast
Eqs. (2) and (4) in terms of $E_{\mathrm{g}}$ :

$$
\begin{aligned}
& \frac{J}{E_{2}-E_{1}}=A \cdot E_{\mathrm{eff}}^{-\gamma} \\
& =\frac{A}{E_{2}-E_{1}} \frac{E_{\mathrm{g}}^{-\gamma+1}}{\gamma-1} \times\left[\left(E_{1} / E_{\mathrm{g}}\right)^{-\gamma+1}-\left(E_{2} / E_{\mathrm{g}}\right)^{-\gamma+1}\right] .
\end{aligned}
$$

We simplify this by setting $r^{2}=E_{2} / E_{1}>1$, from which $E_{1}=E_{\mathrm{g}} / r$ and $E_{2}=r E_{\mathrm{g}}$. Equation (7) then leads to

$\left(\frac{E_{\mathrm{eff}}}{E_{\mathrm{g}}}\right)^{-\gamma}=\frac{1}{\gamma-1} \frac{r^{\gamma-1}-r^{-\gamma+1}}{r-r^{-1}} \rightarrow 1$ as $r \rightarrow 1$.

In order to test how good the geometric-mean approximation is, we take once more the extreme example of $E_{2}=2 E_{1}$ and $\gamma=4$; now we have $r=\sqrt{2}$ and Eq. (8) yields $E_{\text {eff }} / E_{\mathrm{g}}=0.96$; this shows that $E_{\mathrm{g}}$ is a good estimate of $E_{\text {eff }}$ even for this "extreme" case.

We now examine how different measured particle quantities change when the geometric-mean approximation is used for their calculation.

\section{Phase space density with geometric-mean energy}

The differential flux of particles with velocity $\bar{v}$ is given by

$j(E, \Omega) \mathrm{d} E \mathrm{~d} \Omega=f(\overline{\boldsymbol{v}}) v^{3} \mathrm{~d} v \mathrm{~d} \Omega$

where $f(\overline{\boldsymbol{v}}) v^{2}, \mathrm{~d} v \mathrm{~d} \Omega$ is the number of particles per unit volume with velocity between $\left(v_{x}, v_{y}, v_{z}\right)$ and $\left(v_{x}+\mathrm{d} v_{x}\right.$, $\left.v_{y}+\mathrm{d} v_{y}, v_{z}+\mathrm{d} v_{z}\right)$. From the Eq. (9) using relationship $\mathrm{d} E=m v \mathrm{~d} v$ we get the standard relation between differential flux and phase space density

$j=\frac{2 E}{m^{2}} f(\overline{\boldsymbol{v}})$.

The phase space density can be written in units of $\mathrm{km}^{-6} \mathrm{~s}^{3}$ as

$f=m^{2} \frac{j}{E_{\mathrm{eff}}} \cdot 0.53707$,

where $m$ is the particle mass in atomic mass units, $j$ recorded in $\mathrm{cm}^{-2} \mathrm{~s}^{-1} \mathrm{sr}^{-1} \mathrm{keV}^{-1}$ and $E_{\text {eff }}$ the effective energy of the energy channel in keV. Let us test if approximation $E_{\mathrm{eff}}=E_{\mathrm{g}}$ for the phase space density calculations of Cluster/RAPID measurements in Kronberg and Daly (2013) is good enough. Using Eq. (8), the deviation between the power-law phase space density and geometric-mean phase space density is

$\operatorname{dev}_{\mathrm{f}}=f / f_{\mathrm{g}}=E_{\mathrm{g}} / E_{\mathrm{eff}}=\left(\frac{1}{\gamma-1} \frac{r^{\gamma-1}-r^{-\gamma+1}}{r-r^{-1}}\right)^{1 / \gamma}$.

For the previous example of $E_{2}=2 E_{1}$ and $\gamma=4$, the deviation, $\operatorname{dev}_{\mathrm{f}}$ is 1.04 . The difference between RAPID energy channel thresholds is even smaller. 


\subsection{Energy density with geometric-mean energy}

Energy density, $\varepsilon$, for a finite channel should be

$\varepsilon=\int_{E_{1}}^{E_{2}} f E \mathrm{~d}^{3} v$

where $f$ is the phase space density of particles with velocity $v, E_{1}$ and $E_{2}$ are the energy channel thresholds. The energy density expressed through the omnidirectional flux will be the following:

$\varepsilon=\int_{E_{1}}^{E_{2}} \sqrt{\frac{m}{2}} \sqrt{E} j(E) \mathrm{d} E \mathrm{~d} \Omega=2 \pi \sqrt{\frac{m}{2}} \int_{E_{1}}^{E_{2}} \sqrt{E} j(E) \mathrm{d} E$,

where $\Omega$ is the field of view. Here, phase space density, $f$, was converted into differential flux using Eq. (10). Therefore, the simple formula to calculate the energy density for the narrow energy channel will be

$\varepsilon=\pi \sqrt{2 m} \sqrt{E} j(E) \Delta E=\pi \sqrt{2 m} \sqrt{E_{\text {eff }}} j(E) \Delta E$.

The problem which becomes critical in the case of wide energy channels is to decide what value of $E$ to use here. As mentioned in Sect. 3 for defining the effective energy, $E$, the geometric-mean energy, $E_{\mathrm{g}}=\sqrt{E_{1} \cdot E_{2}}$ is often used, rather than a more precise definition from Sect. 2. The question is how reliable is this simplification, at which spectral slopes and energy channel widths is it appropriate to use and how to correct for this.

It is reasonable to assume that at, for example, RAPID energies ( $>30 \mathrm{keV}$ ), the differential flux $j=A \cdot E^{-\gamma}$ has a power law dependence on energy. Therefore,

$$
\begin{aligned}
\varepsilon & =\pi \sqrt{2 m} \int_{E_{1}}^{E_{2}} \sqrt{E} A \cdot E^{-\gamma} \mathrm{d} E \\
& =\pi \sqrt{2 m} \frac{A}{\gamma-3 / 2}\left[E_{1}^{-\gamma+3 / 2}-E_{2}^{-\gamma+3 / 2}\right] .
\end{aligned}
$$

Let us test how well this exact power-law formula compares with the "geometric-mean energy density" found by setting $E \rightarrow E_{\mathrm{g}}$ and $j(E) \rightarrow J /\left(E_{2}-E_{1}\right)$ in Eq. (15). Recall that the measured mean differential flux $J /\left(E_{2}-E_{1}\right)=A \cdot E_{\text {eff }}^{-\gamma}$ (from Eq. 4), is expressed in terms of the effective energy.
Again we use $r^{2}=E_{2} / E_{1}>1$, and then $E_{1}=E_{\mathrm{g}} / r$ and $E_{2}=r E_{\mathrm{g}}$, Eq. (16) will take a form:

$$
\begin{aligned}
\varepsilon= & \pi \sqrt{2 m} \frac{A E_{\mathrm{g}}^{-\gamma+3 / 2}}{\gamma-3 / 2}\left[\left(E_{1} / E_{\mathrm{g}}\right)^{-\gamma+3 / 2}-\left(E_{2} / E_{\mathrm{g}}\right)^{-\gamma+3 / 2}\right] \\
= & \left\{\pi \sqrt{2 m} \sqrt{E_{\mathrm{g}}}\left(A \cdot E_{\mathrm{eff}}^{-\gamma}\right) \Delta E\right\} \\
& \times \frac{1}{\gamma-3 / 2} \frac{E_{\mathrm{g}}}{\Delta E}\left(\frac{E_{\mathrm{g}}}{E_{\mathrm{eff}}}\right)^{-\gamma}\left(r^{\gamma-3 / 2}-r^{-\gamma+3 / 2}\right) \\
= & \left\{\pi \sqrt{2 m} \sqrt{E_{\mathrm{g}}}(J / \Delta E) \Delta E\right\} \\
& \times \frac{1}{\gamma-3 / 2}\left(\frac{E_{\mathrm{g}}}{E_{\mathrm{eff}}}\right)^{-\gamma}\left(\frac{r^{\gamma-3 / 2}-r^{-\gamma+3 / 2}}{r-r^{-1}}\right) \\
= & \varepsilon_{\mathrm{g}} \frac{1}{\gamma-3 / 2}\left(\frac{E_{\mathrm{g}}}{E_{\mathrm{eff}}}\right)^{-\gamma}\left(\frac{r^{\gamma-3 / 2}-r^{-\gamma+3 / 2}}{r-r^{-1}}\right) .
\end{aligned}
$$

Here $\varepsilon_{\mathrm{g}}$ is the geometric-mean energy density, from Eq. (15) with $E_{\mathrm{g}}$ in place of $E=E_{\text {eff }}$ and $J / \Delta E$ for $j(E)$.

We now apply Eq. (8) and get the deviation $\operatorname{dev}_{\varepsilon}$ between the power-law energy density and the geometric-mean energy density:

$\operatorname{dev}_{\varepsilon}=\varepsilon / \varepsilon_{\mathrm{g}}=\frac{\gamma-1}{\gamma-3 / 2} \frac{r^{\gamma-3 / 2}-r^{-\gamma+3 / 2}}{r^{\gamma-1}-r^{-\gamma+1}}$.

Using our previous example of $E_{2}=2 E_{1}$ and $\gamma=4$, Eq. (18) yields $\operatorname{dev}_{\varepsilon}=0.949$.

Note that $r>1$ and ideally $r \rightarrow 1$ for a narrow channel. In this case $\operatorname{dev}_{\varepsilon} \rightarrow 1$. However, in case of wide energy channels or/and soft energy spectra one has to use the formula (Eq. 18) for calculation of the energy density deviation.

\section{Example}

In study of the ion abundance $\left(\mathrm{O}^{+} / \mathrm{H}^{+}\right)$in the terrestrial magnetosphere by Kronberg et al. (2012) one of the aims was a comparison of Cluster/RAPID results to those from other missions. The energy density is commonly used for calculations of the abundance ratios. Therefore, it was necessary to transfer the intensities used in the Kronberg et al. (2012) study in to energy density. There were doubts if one could use the geometric-mean approximation in this case as the width of the energy channels is large. Also protons and oxygen have different spectral slopes. Using Eq. (18) we are able to demonstrate the impact of this approximation.

We take an arbitrary plasma sheet crossing by Cluster SC4, for example, from 11:30 to 24:00 UT on 2 September 2007 and the aim is to calculate the mean energy density ratio, $\varepsilon_{\mathrm{O}^{+}} / \varepsilon_{\mathrm{H}^{+}}$.

First, we calculate the mean values of the ion intensity at different energies during this plasma sheet crossing and from that we derive the spectral characteristics, namely, the effective energy for protons and oxygen and the spectral index $\gamma$ using the method described in Sect. 2, in particular formulas (Eqs. 5 and 6). The same is done using the geometric-mean 
Table 1. Energy spectral index $\gamma$, effective energy, deviations of phase space density and energy density.

\begin{tabular}{lllrrrrrrr}
\hline Species & Energy range 1 & Energy range 2 & $\gamma / \gamma_{\mathrm{g}}$ & $E_{\text {eff1 }} / E_{\mathrm{g} 1}$ & $E_{\text {eff2 }} / E_{\mathrm{g} 2}$ & $f 1 / f 1_{\mathrm{g}}$ & $\varepsilon 1 / \varepsilon 1_{\mathrm{g}}$ & $f 2 / f 2_{\mathrm{g}}$ & $\varepsilon 2 / \varepsilon 2_{\mathrm{g}}$ \\
\hline Protons & $160-374 \mathrm{keV}$ & $374-962 \mathrm{keV}$ & $4.86 / 4.77$ & $225 / 245$ & $546 / 600$ & 1.08 & 0.91 & 1.1 & 0.89 \\
Oxygen & $274-414 \mathrm{keV}$ & $498-638 \mathrm{keV}$ & $3.28 / 3.32$ & $334 / 337$ & $562 / 564$ & 1.01 & 0.98 & 1.003 & 0.99 \\
\hline
\end{tabular}

approximation. For the protons we used the energy channels at $160-374$ and $374-962 \mathrm{keV}$ and for the oxygen at 274 414 and $498-638 \mathrm{keV}$. The deviations of the phase space density and the energy density between those two methods (from Eqs. 12 and 18) are also calculated. The obtained values are listed in Table 1.

The geometric mean is a good approximation as the difference between two methods for all quantities is less than $10 \%$. An exception is the $11 \%$ deviation of the proton energy density which is seen for the widest RAPID energy channel with $E_{1}=374$ and $E_{2}=962 \mathrm{keV}(r=1.6)$.

In order to compare the proton and oxygen observations, which do not have the same energy range, we construct from channels above a wide energy channel with the same width for both species (274- 955 keV), see detailed description in Kronberg et al. (2012).

Using Eq. (15) and the assumption that the effective energy is calculated as the geometric mean, the energy density ratio of oxygen to protons will become a simple relation:

$\frac{\varepsilon_{\mathrm{O}^{+}}}{\varepsilon_{\mathrm{H}^{+}}}=\frac{\pi \sqrt{2 m_{\mathrm{O}}} \cdot \sqrt{E_{\mathrm{eff}}} \cdot J^{\mathrm{O}^{+}} \Delta E}{\pi \sqrt{2 m_{\mathrm{H}}} \cdot \sqrt{E_{\mathrm{eff}}} \cdot J^{\mathrm{H}^{+}} \Delta E}=\frac{4 \cdot J^{\mathrm{O}^{+}}}{J^{\mathrm{H}^{+}}}$.

However, the energy channels are wide, the spectral index $\gamma$ is different for oxygen and protons (in this case it is not clear if one can use Eq. (19)), or the energy density ratio $\varepsilon_{\mathrm{O}^{+}} / \varepsilon_{\mathrm{H}^{+}}$ calculated in this way will significantly deviate from those which are based on the geometric-mean assumption.

Assuming that the proton and oxygen distributions for the constructed wide energy channel have the same spectral slopes as derived above, we can estimate the deviation of the effective energy from the geometric-mean energy. Effective energy for the protons is $430 \mathrm{keV}$, for the oxygen $479 \mathrm{keV}$. These are $\sim 15 \%$ and $\sim 8 \%$ different from the geometricmean energy, which is $511 \mathrm{keV}$. The corresponding energy spectra with denoted effective energies are shown in Fig. 1. The phase space density deviation $f / f_{\mathrm{g}}$ is 1.17 and 1.08 for protons and oxygen, respectively. The energy density deviation $\varepsilon / \varepsilon_{\mathrm{g}}$ is 0.83 and 0.89 for protons and oxygen, respectively. The deviation of the energy density ratio $\varepsilon_{\mathrm{O}^{+}} / \varepsilon_{\mathrm{H}^{+}}$due to the different spectral slope of proton and oxygen in this example is $6 \%$.

Assuming the range of $\gamma=2-4.5$ for $\mathrm{O}^{+}$and $\gamma=3.5-6.5$ for $\mathrm{H}^{+}$we get the spread of the deviations of the energy density ratio $\mathrm{O}^{+} / \mathrm{H}^{+}$up to $19 \%$ with the mean value $\sim 7 \%$ which is less than the statistical error bar of the RAPID measurements. Therefore, the Eq. (19) can be used as an approximation of the energy density ratio $\varepsilon_{\mathrm{O}^{+}} / \varepsilon_{\mathrm{H}^{+}}$. For this spread

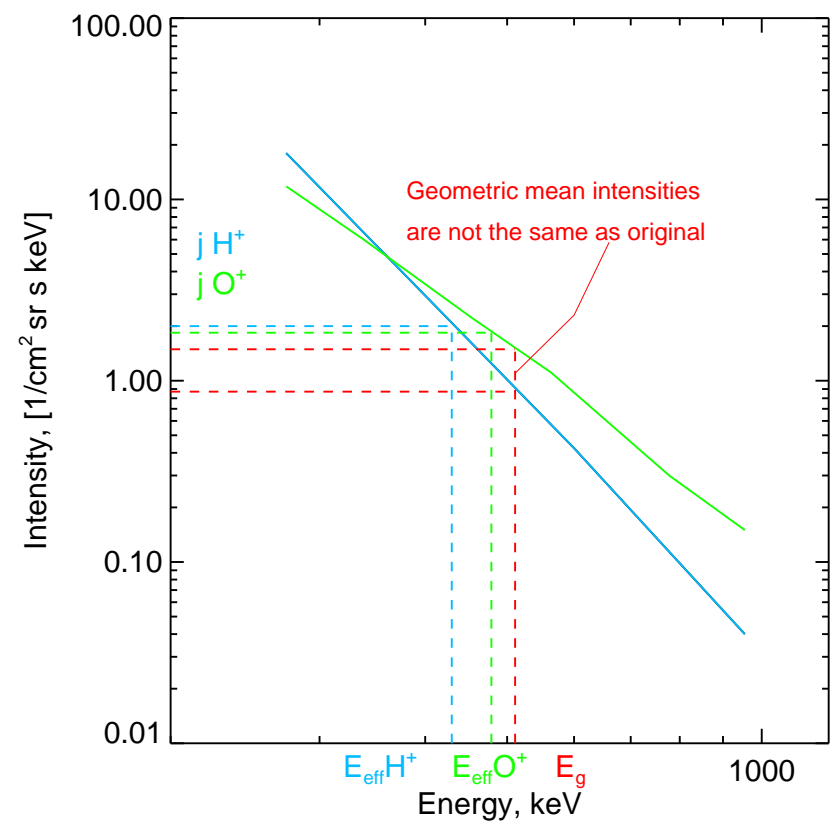

Fig. 1. Mean energy spectra $(274-955 \mathrm{keV})$ for the protons and oxygen for the time period from 11:30 to 24:00 UT on 2 September 2007, observed by Cluster, SC4. The effective energy, $E_{\mathrm{g}}$, based on geometric-mean assumption and its corresponding proton and oxygen intensities are denoted by red colour. There is a mismatch between original intensities and intensities which correspond to the geometric-mean effective energy.

of $\gamma$, the energy density deviates $\sim 12-20 \%$ for protons and $\sim 5-16 \%$ for oxygen. The phase space density deviates $\sim 9$ $25 \%$ for protons and $\sim 0-15 \%$ for oxygen.

\section{Conclusions}

The geometric-mean approximation is a very good approximation of the effective energy at the power law spectral slopes. This can be used for the calculation of phase space density and energy density from Cluster/RAPID ion measurements with prescribed energy thresholds (Daly and Kronberg, 2010). The derived correction is needed for rather extraordinary cases of an energy channel width (as e.g. for merged RAPID channels) and soft spectral slope. 
Acknowledgements. We are grateful for the support and motivation Cluster Active Archive (CAA). We thank the Deutsches Zentrum für Luft und Raumfahrt (DLR) for supporting the RAPID instrument at MPS under grant 50 OC 1101 .

Edited by: M. Taylor

\section{References}

Daly, P. W. and Kronberg, E. A.: RAPID Products at the Cluster Active Archive, in: The Cluster Active Archive, Studying the Earth's Space Plasma Environment, edited by: Laakso, H., Taylor, M., and Escoubet, C. P., Springer, Dordrecht, Heidelberg, London, New York, 145-158, doi:10.1007/978-90-4813499-1_9, 2010.

Escoubet, C. P., Schmidt, R., and Goldstein, M. L.: Cluster - Science and Mission Overview, Space Sci. Rev., 79, 11-32, 1997.

Kronberg, E. A. and Daly, P. W.: Calibration Report of the RAPID Measurements in the Cluster Active Archive (CAA), Tech. Rep. CAA-EST-CR-RAP, available at: http://caa.estec. esa.int/documents/CR/CAA_EST_CR_RAP_v30.pdf, last access: 20 August 2013.
Kronberg, E. A., Haaland, S. E., Daly, P. W., Grigorenko, E. E., Kistler, L. M., Fränz, M., and Dandouras, I.: Oxygen and hydrogen ion abundance in the near-Earth magnetosphere: Statistical results on the response to the geomagnetic and solar wind activity conditions, J. Geophys. Res. Space, 117, A12208, doi:10.1029/2012JA018071, 2012.

Wilken, B., Daly, P. W., Mall, U., Aarsnes, K., Baker, D. N., Belian, R. D., Blake, J. B., Borg, H., Büchner, J., Carter, M., Fennell, J. F., Friedel, R., Fritz, T. A., Gliem, F., Grande, M., Kecskemety, K., Kettmann, G., Korth, A., Livi, S., McKenna-Lawlor, S., Mursula, K., Nikutowski, B., Perry, C. H., Pu, Z. Y., Roeder, J., Reeves, G. D., Sarris, E. T., Sandahl, I., Søraas, F., Woch, J., and Zong, Q.-G.: First results from the RAPID imaging energetic particle spectrometer on board Cluster, Ann. Geophys., 19, 1355-1366, doi:10.5194/angeo-19-1355-2001, 2001. 